\title{
Can the adverse childhood experiences (ACEs) checklist be utilized to predict emergency department visits among children and adolescents?
}

Asmita Bhattarai ${ }^{1,2^{*}}$ D, Gina Dimitropoulos ${ }^{2,3}$, Brian Marriott ${ }^{3,4}$, Jaime Paget ${ }^{4}$, Andrew G. M. Bulloch ${ }^{1,2,5}$, Suzanne C. Tough ${ }^{1,6}$ and Scott B. Patten ${ }^{1,2,5}$

\begin{abstract}
Background: Extensive literature has shown an association of Adverse Childhood Experiences (ACEs) with adverse health outcomes; however, its ability to predict events or stratify risks is less known. Individuals with mental illness and ACE exposure have been shown to visit emergency departments (ED) more often than those in the general population. This study thus examined the ability of the ACEs checklist to predict ED visits within the subsequent year among children and adolescents presenting to mental health clinics with pre-existing mental health issues.

Methods: The study analyzed linked data $(n=6100)$ from two databases provided by Alberta Health Services (AHS). The Regional Access and Intake System (RAIS 2016-2018) database provided data on the predictors (ACE items, age, sex, residence, mental health program type, and primary diagnosis) regarding children and adolescents (aged 0-17 years) accessing addiction and mental health services within Calgary Zone, and the National Ambulatory Care Reporting System (NACRS 2016-2019) database provided data on ED visits. A 25\% random sample of the data was reserved for validation purposes. Two Least Absolute Shrinkage and Selection Operator (LASSO) logistic regression models, each employing a different method to tune the shrinkage parameter lambda (namely cross-validated and adaptive) and performing 10-fold cross-validation for a set of 100 lambdas in each model were examined.

Results: The adaptive LASSO model had a slightly better fit in the validation dataset than the cross-validated model; however, it still demonstrated poor discrimination (AUC 0.60, sensitivity 37.8\%, PPV 49.6\%) and poor calibration (over-triaged in low-risk and under-triaged in high-risk subgroups). The model's poor performance was evident from an out-of-sample deviance ratio of -0.044 .
\end{abstract}

\footnotetext{
* Correspondence: asmita.bhattarai1@ucalgary.ca

'Department of Community Health Sciences, Cumming School of Medicine, University of Calgary, 3280 Hospital Drive NW, Calgary, AB T2N4Z6, Canada

${ }^{2}$ Mathison Centre for Research \& Education, University of Calgary, 3280

Hospital Drive NW, Calgary, AB T2N4Z6, Canada

Full list of author information is available at the end of the article
}

(C) The Author(s). 2021 Open Access This article is licensed under a Creative Commons Attribution 4.0 International License, which permits use, sharing, adaptation, distribution and reproduction in any medium or format, as long as you give appropriate credit to the original author(s) and the source, provide a link to the Creative Commons licence, and indicate if changes were made. The images or other third party material in this article are included in the article's Creative Commons licence, unless indicated otherwise in a credit line to the material. If material is not included in the article's Creative Commons licence and your intended use is not permitted by statutory regulation or exceeds the permitted use, you will need to obtain permission directly from the copyright holder. To view a copy of this licence, visit http://creativecommons.org/licenses/by/4.0/ The Creative Commons Public Domain Dedication waiver (http://creativecommons.org/publicdomain/zero/1.0/) applies to the data made available in this article, unless otherwise stated in a credit line to the data. 
Conclusion: The ACEs checklist did not perform well in predicting ED visits among children and adolescents with existing mental health concerns. The diverse causes of ED visits may have hindered accurate predictions, requiring more advanced statistical procedures. Future studies exploring other machine learning approaches and including a more extensive set of childhood adversities and other important predictors may produce better predictions. Furthermore, despite highly significant associations being observed, ACEs may not be deterministic in predicting health-related events at the individual level, such as general ED use.

Keywords: ACEs, Adverse childhood experiences, Emergency department visit, Prediction, Least absolute shrinkage and selection operator, Mental illness

\section{Background}

Adverse childhood experiences (ACEs) refer to a set of stressors during childhood [1,2]. The first ACE study measured ten types of childhood adversities experienced by an individual before 18 years of age, namely physical abuse, emotional abuse, sexual abuse, emotional neglect, physical neglect, witnessing interpersonal violence, parental divorce, parental substance abuse, living with a family member with mental illness, and incarceration of a family member [1]. The ACEs have been used as a checklist or a tool to measure exposure to childhood adversities by subsequent studies [3-5] and might also have usefulness in clinical application in the context of therapeutic dialogue and trauma-informed care [6-9].

With various studies reporting the prevalence of at least one ACE to be over 50\% [1, 10], ACEs are commonplace. These adversities are associated with poor health and well-being throughout life, the adverse outcomes ranging from smoking, alcoholism, drug abuse, suicide attempts, chronic diseases, mental illness, and even mortality $[1,3-5,11]$. Most of these ACEassociated adverse health conditions are common reasons for people to access acute health care services [12], notably, increased uptake of emergency department (ED) services [11, 13-17]. For instance, one Canadian study reported that, compared to the general population, people with more than one ACE, visit the emergency department up to $29 \%$ more often than those without the exposure [10]. Another study among children and adolescents reported that those who reported childhood abuse had a higher number of ED visits than their counterparts $(2.1$ vs. $1.5, p$-value $<0.01)$ [18]. Individuals with mental illness are also more likely to visit the ED more frequently than those without mental illness [11]. Studies have reported that as many as $25-30 \%$ of the ED visitors have been noted to have a mental illness $[19,20]$. Both ACE exposure and mental illnesses can contribute to overcrowding in emergency departments and place administrative and economic burdens on the health system [11, 19-21].

Preventing ED visits may be a means of reducing medical costs $[13,17,22,23]$, which is the inherent interest of health care reformers and policymakers since those resources could be directed elsewhere to achieve more efficient and equitable service delivery [24]. It has been reported that youth with childhood maltreatment have higher health care utilization costs, and increased ED visits contribute in part to this higher overall cost [25]. Accessing acute health care may indicate positive healthseeking behavior; however, if it is recurrent and is preventable with primary care, it may reflect ineffectiveness in care pathways; especially in educating patients regarding the importance of accessing primary care, assisting them with scheduling appointments, and following them up to monitor them adequately [13, 17, 26, 27].

ACE data has been used in public health programs for various health promotion and illness prevention efforts [28] and is being routinely collected in various health service programs across Canada. However, clinicians and researchers question whether it can be used to predict salient health care events such as future ED visits at the individual level. Being statistically associated with health care outcomes is not the same as being predictive of those outcomes. A significant association of ACEs with ED does not confirm that outcomes can be accurately predicted [29]. An ability to predict this outcome at the individual level could help implement targeted interventions to improve clinical care and reduce emergency visits and unplanned readmissions $[13,14,17,30]$. If childhood adversities are as strongly associated with adverse health outcomes such as suicidal behavior, sexually transmitted infections, problematic substance use in youth and adults as reported by studies [1-3], then they may be useful to predict ED visits. Then, the health care providers would be able to utilize predictions to improve outpatient health services' effectiveness and reduce the burden to emergency and inpatient services [10, 13]. The health service utilization data collected in the Canadian health care system, known as administrative databases (since the data are used in the administration of health services), provides us the opportunity to link the ACE data with prospective follow-up of ED visits data and to evaluate the predictive ability of ACE items, combined with other routinely collected data elements, in predicting the risk of ED visits [31]. The results may contribute to advance the field towards targeted preventive care and, in turn, reduce health care costs. 
Machine learning approaches are gaining more attention as a tool for prediction in research and clinical practice. Many studies have reported that machine learning can better predict patient outcomes (provide more stable predictions) than traditional modeling approaches based on non-regularized regression in various disease conditions and settings [32-35]. These algorithms are even more applicable in health care data since they can account for the interactions, unusual distributions, and a large number of variables [34, 36]. Researchers and clinicians interested in using ACEs data to predict health outcomes have mostly relied on traditional modeling approaches and focused on psychopathological and behavioral outcomes. There is not much evidence around the predictive ability of childhood adversities for important health-related events such as ED visits, irrespective of the prediction modeling approaches (i.e., traditional and machine learning). Studies examining the characteristics of frequent ED users have also failed to examine childhood adversities as potential risk factors for increased ED visits [37]. Hence, it is worth exploring whether a machine learning approach can lead to a prediction that is accurate enough to support clinical decisions and build strategies to intervene in advance to avoid future adverse health events such as ED visits [32, 38, 39]. The least absolute shrinkage and selection operator (LASSO) models are gaining popularity in prediction since they have better interpretability than other less transparent machine learning approaches $[35,40]$. The models are developed in a training dataset followed by testing or validation in a new dataset. Also, through regularization (shrinkage), LASSO is believed to reduce overfitting while dealing with many predictors [40], leading to strong prediction performance.

This study aims to address the existing knowledge gap and provide clinicians a potential resource to guide their clinical decision-making by using the routinely collected and readily available patient information. The study thus examined the ability of specific childhood adversities, measured using the ACE checklist, in predicting the risk of ED visits within the subsequent year among children and adolescents with mental illness presenting to outpatient addiction and mental health services program. We employed LASSO as a machine learning modeling approach.

\section{Objective}

The objective of the study was to evaluate the predictive performance of the machine learning model incorporating $\mathrm{ACEs}$ and routinely available variables to predict subsequent one-year ED visits among children and adolescents seen for pre-existing addiction or mental health concerns in an outpatient setting.

\section{Methods}

\section{Data source}

The data was available through record linkage of two sizable databases, namely, Regional Access and Intake System (RAIS; a local health service registration database) and National Ambulatory Care Repository System (NACRS; a national health administrative database). The RAIS database, developed as a client tracking system to support the Alberta Health Services' (AHS) Child and Adolescent Addiction and Mental Health and Psychiatry Program (CAAMHPP), provided information on multiple predictors $[41,42]$. The CAAMHPP is a regional program serving a geographically defined catchment area, i.e., Calgary Zone in Alberta, and consists of several services, including inpatient, outreach, community and outpatient, day programs, school-based services, and specialized services. These services are typically provided for more complex/severe clients requiring a higher level of addiction or mental health service versus being treated by a community agency or family physicians.

In addition to demographic and clinical information, data on ACEs have been collected routinely since 2016 from a cohort of children and adolescents accessing addiction and mental health services within Calgary by CAAM HPP across its continuum of care [9]. The ACE questionnaire is administered by CAAMHPP clinicians using the ACE checklist through a face-to-face interview with children/adolescents aged below 18 years who seek specialty mental health care or with the parents if the children could not respond [42]. Preschool-aged children were also included in the study, and their ACE data were collected from the parents or caregivers. There is no predefined cut-off to determine whether the children or their parents would be the informant, and hence the clinicians administer it based on their clinical judgment. Informed consent was obtained from parents/guardians for the children below 18 years of age. The details about the ACE survey at CAAMHPP, such as data collection, storage, staff training, are described elsewhere [43]. The prospective follow-up of subsequent ED visits was available from the NACRS, a clinical, administrative database that collects all ambulatory care data, including ED visits within Canada [27]. The linkage was done using a common identifier, Personal Health Number (PHN), and the final analysis was performed using the linked and de-identified dataset.

The sample was comprised of children and adolescents aged 0 to 17 years who have enrolled in the AHS's CAAMHPP program (intensive inpatient and outpatient mental health services) between June 2016 to June 2018 and all-cause ED visits during the year following their first registration. The end of the follow-up period was 30 June 2019. The linkage resulted in 6100 unique observations and 2545 matched ED visits within the year following enrollment. 


\section{Inclusion and exclusion criteria}

The study included all the patients aged $0-17$ years registered in the RAIS database from June 2016 to June 2018. A single patient might have had multiple enrolments into the CAAMHPP program, but we included only the first enrolment since it is clinically more relevant to predict future outcomes at the point of the first contact with the program. Hence the ACE data used for prediction was the very first $\mathrm{ACE}$ assessment during the first enrollment. Also, a person might have had multiple ED visits, but we included only the index visit as per our operational definition. The ED visit on the same day of enrollment into the program was not considered an outcome because such predictions would not be useful for prevention.

\section{Measures}

Predictors

ACE items The 10 ACE items, namely exposure to physical abuse, witnessing inter-parental violence, parental divorce, parental substance use, emotional abuse, emotional neglect, physical neglect, incarcerated family member, and living with a mentally ill family member [9], were included as factor variables (yes/no/missing).

Demographic and clinical variables Age at program enrollment was included as a continuous variable. Other categorical variables were reclassified and included as factor variables, which were namely sex (male; female; and neither male nor female), residence (urban; rural/ unclassified or unknown; and missing), and program type (community bed; community clinic; consultation service; day treatment; emergency service; inpatient unit; outpatient service; school-based service; and specialized service). The factor variable primary diagnosis included categories: anxiety, bipolar disorder, depressive disorder, disruptive/impulse control/conduct disorder, feeding/ eating disorder, gender dysphoria, medical diagnosis, neurodevelopmental disorder, obsessive-compulsive disorder, personality disorder, psychosocial factor, schizophrenia/psychotic disorder, somatic symptom, substancerelated disorder, trauma/stressor-related disorder, other mental disorders, and others.

\section{Outcome}

The outcome was an ED visit (yes/no), defined as the index ED visit between 1 day to one-year post-first enrollment into the CAAMHPP services.

\section{Data management}

The encrypted RAIS and NACRS databases were made available by Alberta Health Services (AHS) through a secure data transfer process. The original data was available in Microsoft Excel format. The linkage was performed in Excel using a common identifier, Personal Health Number (PHN). The final linked de-identified dataset with 6100 unique observations (children enrolled in RAIS) matched with 2545 index ED visits (between 1 day to one-year post-enrollment to CAAMHPP) was extracted for analysis. The details of the data management process performed in Excel and STATA before reaching the final sample size from the raw data received is illustrated in the flow diagram (Fig. 1). All the analyses were performed in STATA/SE version 16 [44]. All the methods were carried out in accordance with University of Calgary's guidelines and regulations for conducting research.

\section{Handling of missing data}

The missing values in the predictor variables were less than $10 \%$ of the total sample. We examined the pattern of missing data in predictor variables in relation to the ED visit status (Table 2) and found that the missing data is consistently more among those with an ED visit than their counterparts. Hence, with an assumption of missing not at random (MNAR) and that the missing values may contribute to prediction [45], missing data were analyzed as a separate category. The assumption is well supported by the results (Table 3), i.e., higher odds of ED visits were seen among people not responding compared to those who reported no exposure in most of the predictor variables.

\section{Statistical analysis \\ Descriptive analysis}

The subjects' basic demographic and clinical characteristics were described as percentages and 95\% Confidence Intervals (CIs). The prevalence of specific childhood adversities, all-cause, and mental illness specific index ED visits were also reported as percentages and 95\% CIs. The distribution of the predictors was also stratified by ED visit status.

\section{Measures of association}

The association between childhood adversities and ED visits was examined using logistic regression analysis and the results are reported as Odds Ratios (OR) and 95\% CIs.

\section{Prediction modeling}

Model development The LASSO approach taken is a logistic regression with a regularization approach that incorporates a penalty to the log-likelihood function to shrink imprecise coefficients towards zero, thereby minimizing overfitting. The predictor selection is data driven. LASSO regression allows all predictors in the 


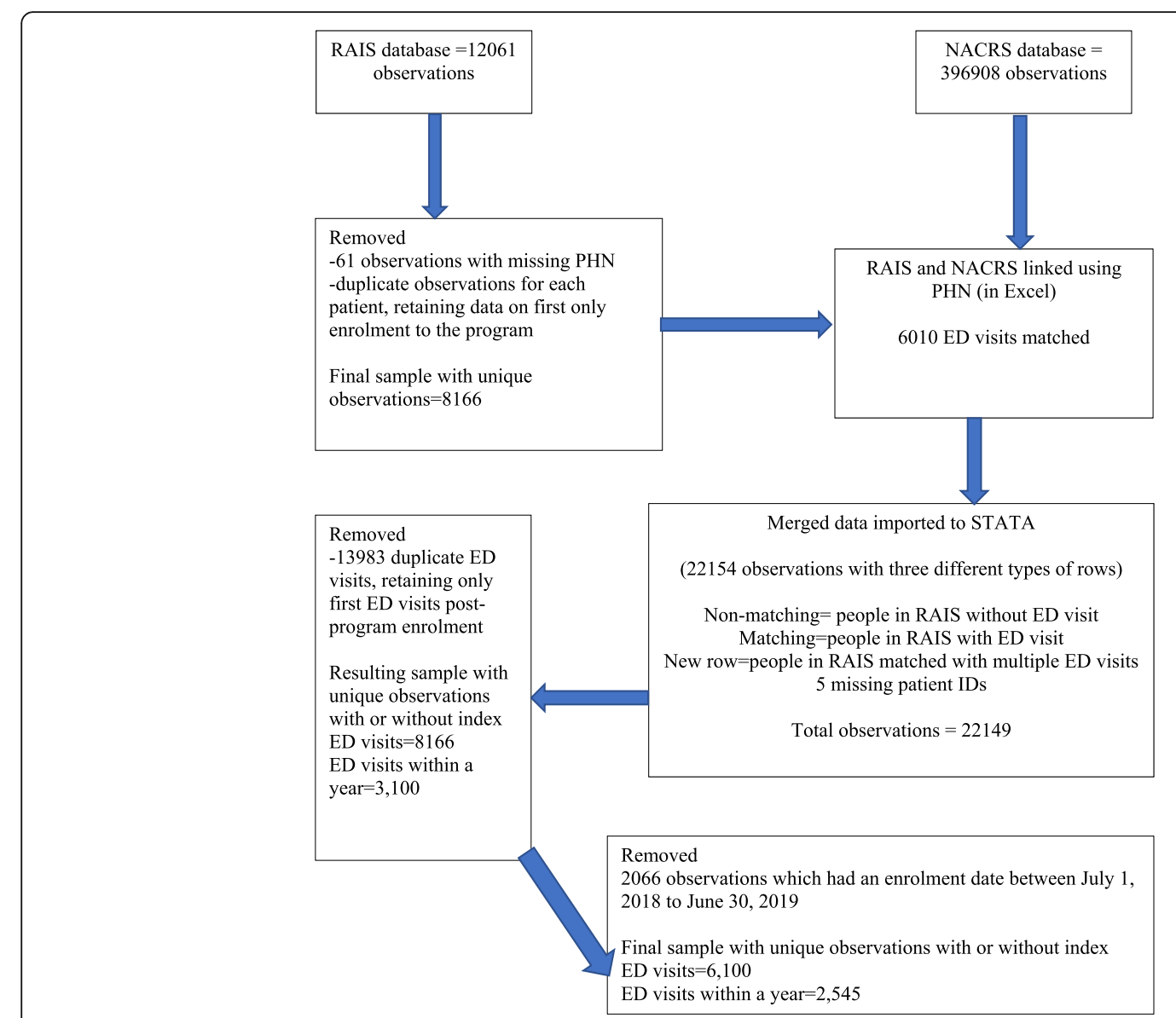

Fig. 1 Derivation of the study sample

dataset in a single model, and predictor selection happens during the model development phase. The algorithms shrink the estimates (odds ratios) towards the null with shrinkage with the threshold for dropping a variable being governed by a single hyperparameter called lambda. Cross-validation (in the current study, 10 -fold cross-validation) is used to identify the lambda value that produced the best-fitting parsimonious model, minimizing the model deviance $[33,45,46]$. LASSO is believed to have a crucial advantage of consistently identifying the true underlying model and effective handling of multicollinearity between predictors enabling navigation of complex associations in health care data [47, 48]. The dataset was split into two randomly selected subsamples containing $75 \%$ and $25 \%$ of the total sample and named as "training" and "validation" samples. Splitting allows for a more accurate determination of the model's performance in a new dataset and reduces overfitting [48]. The training sample was used to develop and internally validate the prediction models, and the validation sample was used to validate the selected prediction models externally.
Using the training data, two LASSO logistic regression models, each employing a different method to tune the shrinkage parameter lambda, namely Cross-validated (CV) and Adaptive, and performing 10-fold crossvalidation for a set of 100 lambdas; were examined. CV is the default method of selecting tuning parameters in LASSO in STATA, which finds the lambda that minimizes the out-of-sample prediction error [49]. The adaptive method is a multistep version of the LASSO, the first step being $\mathrm{CV}$ and the second being $\mathrm{CV}$ among the covariates selected in the first step, also enabling exclusion of some extra variables $[40,44]$. Each item in the ACEs checklist was treated as a separate predictor. ACE ratings have often been evaluated using simple scoring procedures (e.g., the number of reported adversities) using a cut-point based interpretation. However, some adversities are expected to have a greater impact than others, which is neglected in this approach to scoring. Regression-based machine learning techniques are capable of handling more complex data structures as these employ strategies to prevent over-fitting, thereby potentially making better use of the available data [50], and to 
reflect each item's varying contribution. In the logistic regression-based machine learning approach used here, the regression coefficients associated with each adversity allow each one to make a different level of contribution to prediction. In total, ten ACE items, age, sex, residence, clinical diagnosis, program type, and the two-way interaction between each of the variables were included in the models to determine a model with the best predictive performance.

Assessment of model performance The model performance was evaluated using measures of model discrimination and calibration. Discrimination of the model, which enables the model to separate individuals who end up to ER from those who do not, was quantified with sensitivity, specificity, positive and negative predictive value (PPV and NPV), and area under the receiver operating characteristics curve (AUC). The AUC ranges from 0 to 1 , and the higher the AUC (preferably $>0.8$ ), the better the prediction power of the model to be applied clinically $[34,36]$. The resulting confusion matrices were used to visualise and identify the model maximizing correct classification (Accuracy $=($ True positive + True negative) / (True Positive + True Negative + False Positive + False Negative)). The overall average correct classification was also used to assess model performance. However, our goal was to attain a good balance between higher AUC, sensitivity, and PPV. The discrimination was assessed in both training and validation sub-sample, but with greater emphasis on the models' performance in the validation sample.

Calibration of the model was assessed to measure the accuracy by which the model's predicted event rates match the overall observed rates in the validation dataset. The predicted probability of ED visits was divided into four risk categories $(<25 \%, 25-50 \%, 50-75$, and $>$ $75 \%$ ), and the relative frequency of observed events was calculated in each of those risk categories. If the model has good calibration, the empirical probability should be close to the predicted probability [51]. The risk stratification capacity was calculated as the proportion of children and adolescents categorized as low-risk or high-risk [29]. The calibration was also visualized by plotting observed $E D$ visits in the $x$-axis vs. predicted $E D$ visits in the $y$-axis, where the perfect predictions fall along the 45-degree line [45]. Additionally, the models' predictive performance regarding out-of-sample prediction was assessed with an out-of-sample deviance ratio (value usually ranges from 0 to 1 , but can be negative sometimes), which is the goodness of fit statistic. The out-ofsample deviance ratio in logistic LASSO models is analogous to the R-squared (higher the better; preferably more than 0.5 ) in the linear LASSO models, which refers to the proportion of variance in the outcome variable accounted for by the model under evaluation [49].

\section{Subgroup analysis}

Further subgroup analysis in terms of the LASSO models' performance in predicting all-cause ED visits using ACE items was conducted among adolescents (10 to 17 years) separately. This subgroup is important because adolescence is a period that is more vulnerable in terms of ACEs exposure as well as mental illnesses [52]. The preventive care needs, and approaches might also be different between children and adolescents. Also, with the assumption that ACE items and the clinical and demographic information might be better predictive of cause-specific ED visits, we explored the predictive performance for mental health issues-specific ED visits among children and adolescents. All of the diagnoses listed in Table 1 except medical conditions were included as mental health issues. This approach is important because these prediction models' utility is more for the health care providers working with children and adolescents with pre-existing mental health concerns at the CAAMHPP program. They are thus more concerned with and are more equipped to implement strategies that prevent mental illness related ED visits if they could predict such visits ahead of time.

\section{Sensitivity analysis}

There is no consensus on the way ACEs are evaluated, and all the approaches being used have their own value. Apart from the effect of individual ACE items on health, the dose-response relationship between ACEs and adverse health has also been consistently reported in research. The ACEs as a cumulative score examine the adversities as a single construct, irrespective of the specific adversities involved, and address the co-occurrence of such adversities [53]. We examined whether examining ACEs as a cumulative score improves the predictive performance. Also, rather than creating a single cumulative score, some researchers have advocated categorizing the exposure into separate groups based on the types of ACEs, taking into consideration the differential mechanism towards their psychopathological sequelae [54]. Based on the dimensional model of adversity suggested in the existing literature $[53,54]$, we examined the predictive ability of threat based (emotional abuse, sexual abuse, physical abuse, and witnessing intimate partner violence) and deprivation based (emotional neglect, physical neglect, parental divorce, mental illness in family, substance use in family and incarceration of family member) adversities from the ACEs checklist in two separate models. 
Table 1 Distribution of the demographic and clinical characteristics in the full sample and across ED visit status

\begin{tabular}{|c|c|c|c|}
\hline Characteristics & $\begin{array}{l}\text { Full sample } \\
\%(95 \% \mathrm{Cl})\end{array}$ & $\begin{array}{l}\text { ED visit } \\
\%(95 \% \mathrm{Cl})\end{array}$ & $\begin{array}{l}\text { No ED visits } \\
\%(95 \% \mathrm{Cl})\end{array}$ \\
\hline Age in years (mean/SD) & $12.1(4.3)$ & $12.6(4.2)$ & $11.7(4.3)$ \\
\hline \multicolumn{4}{|l|}{ Sex } \\
\hline Male & $44.7(43.5,46.0)$ & $40.4(38.5,42.4)$ & $47.8(46.2,49.4)$ \\
\hline Female & $54.2(53.0,55.5)$ & $58.2(56.3,60.1)$ & $51.4(49.7,53.0)$ \\
\hline Neither male/female & $1.1(0.8,1.4)$ & $1.4(1.0,1.9)$ & $0.8(0.6,1.2)$ \\
\hline \multicolumn{4}{|l|}{ Residence } \\
\hline Rural & $4.8(4.3,5.4)$ & $3.5(2.9,4.3)$ & $5.8(5.0,6.6)$ \\
\hline Urban & $89.5(88.7,90.3)$ & $91.8(90.7,92.8)$ & $87.9(86.8,89.0)$ \\
\hline Unclassified/unknown & $0.8(0.6,1.0)$ & $0.6(0.4,1.0)$ & $0.9(0.6,1.2)$ \\
\hline Missing & $4.9(4.4,5.4)$ & $4.1(3.4,4.9)$ & $5.4(4.7,6.2)$ \\
\hline \multicolumn{4}{|l|}{ Program type } \\
\hline Community bed & $1.3(1.1,1.6)$ & $1.8(1.4,2.4)$ & $1.0(0.7,1.3)$ \\
\hline Community clinic & $22.2(21.2,23.3)$ & $21.6(20.0,23.2)$ & $22.7(21.3,24.1)$ \\
\hline Consultation service & $10.5(9.7,11.3)$ & $10.8(9.7,12.1)$ & $10.2(9.3,11.3)$ \\
\hline Day treatment & $1.4(1.1,1.7)$ & $1.2(0.9,1.7)$ & $1.5(1.2,2.0)$ \\
\hline Emergency service & $14.5(13.6,15.4)$ & $17.6(16.2,19.1)$ & $12.2(11.2,13.3)$ \\
\hline Inpatient unit & $13.7(12.8,14.5)$ & $17.7(16.3,19.3)$ & $10.7(9.8,11.8)$ \\
\hline Outpatient service & $16.3(15.4,17.3)$ & $15.3(14.0,16.8)$ & $17.0(15.8,18.3)$ \\
\hline School-based service & $14.6(13.7,15.5)$ & $9.0(8.0,10.2)$ & $18.5(17.3,19.8)$ \\
\hline Specialized service & $5.6(5.0,6.2)$ & $4.9(4.1,5.8)$ & $6.1(5.3,6.9)$ \\
\hline \multicolumn{4}{|l|}{ Primary diagnosis } \\
\hline Anxiety disorder & $24.3(23.2,25.4)$ & $23.5(21.9,25.1)$ & $24.9(23.5,26.3)$ \\
\hline Bipolar disorder & $0.2(0.1,0.4)$ & $0.2(0.1,0.5)$ & $0.2(0.1,0.4)$ \\
\hline Depressive disorder & $12.5(11.7,13.4)$ & $15.0(13.6,16.4)$ & $10.8(9.8,11.8)$ \\
\hline Disruptive/impulse-control/conduct disorder & $1.5(1.2,1.8)$ & $1.8(1.3,2.4)$ & $1.3(0.9,1.7)$ \\
\hline Feeding/eating disorder & $2.4(2.0,2.8)$ & $2.2(1.7,2.8)$ & $2.5(2.1,3.1)$ \\
\hline Gender dysphoria & $0.2(0.1,0.3)$ & $0.2(0.1,0.5)$ & $0.2(0.1,0.4)$ \\
\hline Medical disorder & $0.7(0.5,0.9)$ & $0.7(0.4,1.1)$ & $0.7(0.5,1.0)$ \\
\hline Neurodevelopmental disorder & $19.5(18.5,20.5)$ & $19.3(17.8,20.9)$ & $19.7(18.4,21.0)$ \\
\hline Obsessive-compulsive disorder & $1.6(1.3,2.0)$ & $1.1(0.8,1.6)$ & $2.0(1.6,2.5)$ \\
\hline Other mental disorders & $4.2(3.8,4.8)$ & $3.3(2.7,4.1)$ & $4.9(4.2,5.7)$ \\
\hline Personality disorder & $0.2(0.1,0.4)$ & $0.2(0.1,0.5)$ & $0.3(0.1,0.5)$ \\
\hline Psychosocial factor & $17.3(16.4,18.3)$ & $14.7(13.4,16.2)$ & $19.1(17.9,20.5)$ \\
\hline Schizophrenia/psychotic disorder & $0.7(0.5,1.0)$ & $0.9(0.6,1.4)$ & $0.6(0.4,0.9)$ \\
\hline Somatic symptom & $0.3(0.2,0.5)$ & $0.6(0.3,0.9)$ & $0.2(0.1,0.4)$ \\
\hline Substance-related disorder & $0.9(0.7,1.2)$ & $1.2(0.9,1.7)$ & $0.7(0.5,1.0)$ \\
\hline Trauma/stress-related disorder & $13.1(12.3,14.0)$ & $14.8(13.5,16.2)$ & $12.0(10.9,13.1)$ \\
\hline Others & $0.2(0.1,0.3)$ & $0.3(0.2,0.6)$ & $0.1(0.0,0.3)$ \\
\hline
\end{tabular}

\section{Results}

\section{Descriptive}

The total sample size in the linked data was 6100 . The description of the cohort of children and adolescents understudy with stratification by ED visit status is presented in Table 1 . The average age of the sample was 12.1 (SD 4.3) years, ranging from 0 to 17 years. The majority of the participants were females (54.2\%), and most resided in urban areas (89.5\%). Anxiety was the most prevalent disorder (24.3\%) at admission to the mental 
health services, followed by neurodevelopmental disorders (19.5\%), psychosocial factors (17.3\%), trauma/stressor-related disorders (13.1\%), and depressive mood disorders (12.5\%). Most of the participants were enrolled from community clinics $(22.2 \%)$, followed by outpatient services (16.3\%), emergency services (14.5\%), and school-based services (14.6\%).

The prevalence of a one-year index all-cause ED visit in the sample was $41.7 \%$ (95\% CI 40.5, 43.0). The mean number of ED visits within a year, among those making at least one ED visit, was 3.4 (SD 4.1) times. Most of the index ED visits were for medical conditions (59.1\%), followed by depressive disorder (10.3\%), trauma-stressrelated disorder (9.9\%), and anxiety disorder (6.0\%). The most-reported ACE items were living with a mentally ill family member (50.0\%) and parental divorce (48.7\%), and the least reported were sexual abuse (6.8\%) and having an incarcerated family member (7.8\%). Compared to those without ED visits, more children and adolescents with ED visits reported exposure to individual ACE items (Table 2).

Most of the ACE items (except incarcerated family members) were significantly associated with index ED visits within a year, as evidenced by a $p$-value of $<0.05$ and non-inclusion of the null value of $\mathrm{OR}=1$ within the 95\% CI. The statistically significant odds ratios ranged from 1.20 to 1.72 , suggesting a moderate association (Table 3).

\section{LASSO logistic regression models}

The summary and goodness-of-fit statistics for the LASSO models in the training data are shown in Table 4. Refer to Additional file 1 for the list of variable combinations with non-zero coefficients retained by the two LASSO models.

\section{Discrimination}

Table 5 represents the discriminative ability of the LASSO models. The average cross-validated AUC, sensitivity, specificity, PPV, NPV, and overall classification accuracy within the training dataset and the same calculated in the external validation dataset are presented. None of the models were significantly superior in their discrimination ability, both having an AUC around 0.6 . This suggests a $60 \%$ probability that a randomly chosen individual with an ED visit will have a higher risk score than a randomly selected individual without the outcome. Using the classification threshold of 0.5 , the CV model achieved a sensitivity of $29.2 \%$, PPV of $52.5 \%$, and overall classification accuracy of $60.9 \%$ in the validation dataset. The adaptive model appeared to have a better balance between AUC, sensitivity, and accuracy. The sensitivity achieved from the adaptive model was $37.8 \%$, the PPV was $49.6 \%$, and the
Table 2 Distribution of reports of ACE items the full sample and across ED visit status

\begin{tabular}{|c|c|c|c|}
\hline Characteristics & $\begin{array}{l}\text { Full sample } \\
\%(95 \% \mathrm{Cl})\end{array}$ & $\begin{array}{l}\text { With ED visit } \\
\%(95 \% \mathrm{Cl})\end{array}$ & $\begin{array}{l}\text { Without ED visit } \\
\%(95 \% \mathrm{Cl})\end{array}$ \\
\hline \multicolumn{4}{|l|}{ Emotional abuse } \\
\hline Yes & $26.5(25.4,27.6)$ & $30.9(29.1,32.7)$ & $23.3(22.0,24.8)$ \\
\hline No & $71.1(70.0,72.2)$ & $66.5(64.6,68.3)$ & $74.4(73.0,75.8)$ \\
\hline Missing & $2.4(2.0,2.8)$ & $2.6(2.1,3.3)$ & $2.2(1.8,2.8)$ \\
\hline \multicolumn{4}{|l|}{ Physical abuse } \\
\hline Yes & $13.6(12.8,14.5)$ & $16.6(15.2,18.1)$ & $11.4(10.4,12.5)$ \\
\hline No & $83.4(82.5,84.3)$ & $80.0(78.4,81.5)$ & $85.9(84.7,87.0)$ \\
\hline Missing & $3.0(2.6,3.4)$ & $3.4(2.8,4.2)$ & $2.7(2.2,3.3)$ \\
\hline \multicolumn{4}{|l|}{ Sexual abuse } \\
\hline Yes & $6.8(6.2,7.5)$ & $8.8(7.8,10.0)$ & $5.4(4.7,6.2)$ \\
\hline No & $89.2(88.4,90.0)$ & $86.2(84.9,87.5)$ & $91.4(90.4,92.2)$ \\
\hline Missing & $3.9(3.5,4.5)$ & $5.0(4.2,5.9)$ & $3.2(2.7,3.8)$ \\
\hline \multicolumn{4}{|c|}{ Emotional neglect } \\
\hline Yes & $29.9(28.7,31.0)$ & $34.3(32.5,36.2)$ & $26.7(25.3,28.2)$ \\
\hline No & $67.1(65.9,68.3)$ & $62.9(61.0,64.7)$ & $70.1(68.6,71.6)$ \\
\hline Missing & $3.0(2.6,3.5)$ & $2.8(2.2,3.5)$ & $3.2(2.7,3.8)$ \\
\hline \multicolumn{4}{|l|}{ Physical neglect } \\
\hline Yes & $11.2(10.5,12.0)$ & $12.7(11.5,14.1)$ & $10.2(9.2,11.2)$ \\
\hline No & $86.3(85.4,87.2)$ & $84.6(83.1,85.9)$ & $87.6(86.4,88.6)$ \\
\hline Missing & $2.4(2.1,2.9)$ & $2.7(2.1,3.4)$ & $2.3(1.8,2.8)$ \\
\hline \multicolumn{4}{|l|}{ Parents divorced } \\
\hline Yes & $48.7(47.5,50.0)$ & $51.6(49.7,53.6)$ & $46.6(45.0,48.3)$ \\
\hline No & $50.3(49.1,51.6)$ & $47.2(45.3,49.2)$ & $52.6(50.9,54.2)$ \\
\hline Missing & $0.9(0.7,1.2)$ & $1.1(0.8,1.6)$ & $0.8(0.5,1.1)$ \\
\hline \multicolumn{4}{|c|}{ Interpersonal violence in family } \\
\hline Yes & $19.4(18.4,20.4)$ & $21.9(20.3,23.5)$ & $17.6(16.3,18.8)$ \\
\hline No & $76.5(75.4,77.5)$ & $73.2(71.5,74.9)$ & $78.8(77.4,80.1)$ \\
\hline Missing & $4.1(3.7,4.7)$ & $4.9(4.1,5.8)$ & $3.6(3.1,4.3)$ \\
\hline \multicolumn{4}{|c|}{ Substance use in family } \\
\hline Yes & $25.0(24.0,26.1)$ & $27.0(25.3,28.8)$ & $23.6(22.2,25.0)$ \\
\hline No & $70.7(69.5,71.8)$ & $67.9(66.1,69.7)$ & $72.7(71.2,74.2)$ \\
\hline Missing & $4.3(3.8,4.8)$ & $5.1(4.3,6.0)$ & $3.7(3.1,4.4)$ \\
\hline \multicolumn{4}{|c|}{ Mental illness in family } \\
\hline Yes & $50.0(48.7,51.2)$ & $52.3(50.3,54.2)$ & $48.3(46.7,50.0)$ \\
\hline No & $46.5(45.2,47.7)$ & $43.7(41.8,45.7)$ & $48.4(46.8,50.1)$ \\
\hline Missing & $3.6(3.1,4.1)$ & $4.0(3.3,4.8)$ & $3.3(2.7,3.9)$ \\
\hline \multicolumn{4}{|c|}{ Family member in prison } \\
\hline Yes & $7.8(7.1,8.5)$ & $7.9(6.9,9.0)$ & $7.7(6.9,8.6)$ \\
\hline No & $86.3(85.4,87.1)$ & $84.7(83.3,86.1)$ & $87.4(86.3,88.5)$ \\
\hline Missing & $5.9(5.4,6.6)$ & $7.4(6.5,8.5)$ & $4.9(4.2,5.6)$ \\
\hline
\end{tabular}


Table 3 Association between ACE items and index ED visit within a year of enrolment to mental health services

\begin{tabular}{|c|c|c|c|}
\hline ACE items & Odds Ratio (OR) & $95 \% \mathrm{Cl}$ & $p$-value \\
\hline \multicolumn{4}{|c|}{ Emotional abuse } \\
\hline Yes & 1.48 & $1.32,1.66$ & $<0.001$ \\
\hline No & Ref & Ref & Ref \\
\hline Missing & 1.33 & $0.95,1.85$ & 0.09 \\
\hline \multicolumn{4}{|c|}{ Physical abuse } \\
\hline Yes & 1.56 & $1.35,1.81$ & $<0.001$ \\
\hline No & Ref & Ref & Ref \\
\hline Missing & 1.37 & $1.02,1.85$ & 0.03 \\
\hline \multicolumn{4}{|c|}{ Sexual abuse } \\
\hline Yes & 1.72 & $1.41,2.10$ & $<0.001$ \\
\hline No & Ref & Ref & Ref \\
\hline Missing & 1.64 & $1.26,2.12$ & $<0.001$ \\
\hline \multicolumn{4}{|c|}{ Emotional neglect } \\
\hline Yes & 1.43 & $1.28,1.60$ & $<0.001$ \\
\hline No & Ref & Ref & Ref \\
\hline Missing & 0.97 & $0.72,1.31$ & 0.8 \\
\hline \multicolumn{4}{|c|}{ Physical neglect } \\
\hline Yes & 1.30 & $1.11,1.52$ & 0.001 \\
\hline No & Ref & Ref & Ref \\
\hline Missing & 1.21 & $0.88,1.68$ & 0.2 \\
\hline \multicolumn{4}{|c|}{ Parents divorced } \\
\hline Yes & 1.23 & $1.11,1.37$ & $<0.001$ \\
\hline No & Ref & Ref & Ref \\
\hline Missing & 1.61 & $0.95,2.72$ & 0.07 \\
\hline \multicolumn{4}{|c|}{ Interpersonal violence in family } \\
\hline Yes & 1.34 & $1.18,1.53$ & $<0.001$ \\
\hline No & Ref & Ref & Ref \\
\hline Missing & 1.44 & $1.12,1.86$ & 0.004 \\
\hline \multicolumn{4}{|c|}{ Substance use in family } \\
\hline Yes & 1.23 & $1.09,1.38$ & 0.001 \\
\hline No & Ref & Ref & Ref \\
\hline Missing & 1.47 & $1.15,1.89$ & 0.002 \\
\hline \multicolumn{4}{|c|}{ Mental illness in family } \\
\hline Yes & 1.20 & $1.08,1.33$ & 0.001 \\
\hline No & Ref & Ref & Ref \\
\hline Missing & 1.36 & $1.03,1.79$ & 0.02 \\
\hline \multicolumn{4}{|c|}{ Incarceration of family member } \\
\hline Yes & 1.05 & $0.87,1.27$ & 0.6 \\
\hline No & Ref & Ref & Ref \\
\hline Missing & 1.57 & $1.27,1.95$ & $<0.001$ \\
\hline
\end{tabular}

overall classification accuracy was $59.5 \%$. We saw a slightly weaker performance of the CV model in the validation dataset (AUC in training dataset $0.680-0.698$ vs. AUC in validation dataset $0.610-0.604$ ), which is expected. However, the large difference between the AUCs in training and validation datasets (0.698 vs. 0.604) in the adaptive model suggests that the model was overfitting. We set different classification thresholds to see whether the models attain better discrimination than those with a threshold of 0.5 ; however, there were no meaningful improvements (results not shown).

The two models' discrimination is illustrated by the graph of ROC curves (plotted as sensitivity vs. (1-specificity)) in Fig. 2. Both the curves, i.e., from the $\mathrm{CV}$ and adaptive model, were close to the reference line.

\section{Calibration}

The calibration performance of the models in the validation dataset is represented in the calibration matrix in Table 6. A good calibration refers to less variability between the observed and predicted rates of ED visits. It is also more acceptable if the model under-triages low-risk individuals than if it under triages high-risk individuals. In the adaptive model, the observed rate and 95\% CI (39.8, $95 \%$ CI 36.5, 43.1) fell within the 25-49\% risk category, whereas there was over-triage in the low risk (predicted probability $<25 \%$ ) category and under-triage in the high risk (predicted probability $>50 \%$ ) categories. The variability was highest in the $75-100 \%$ risk category, observed ED visits $43.1 \%$ (95\% CI 32.0, 54.9). In the CV model, the observed rates and corresponding 95\% CI fell within the < $25 \%$ and $25-49 \%$ risk categories but failed to do so in the higher-risk categories where there was evidence of undertriage. The models assigned a small number of predicted probabilities for the highest and lowest risk children and adolescents. The adaptive model had slightly higher risk stratification capacity in the lowest and highest risk categories (4.2 and 15.7\%, respectively). The out-of-sample deviance ratio of the $\mathrm{CV}$ and adaptive models were far less than 1 (0.0246 and -0.0448 , respectively) (Table 4).

The LASSO models' calibration performance is illustrated by the observed versus predicted values plot in Fig. 3 (A, B). There is good calibration if the model predictions curve is closer to the perfect prediction line. The plot for the CV model (Fig. 3A) shows that the predicted probabilities are lower on average than observed, especially in the low and high-risk levels. The adaptive model plot (Fig. 3B) shows that the predicted probabilities are higher than those observed in lower-risk groups and lower than those observed in higher-risk groups.

Since none of the models had a good predictive performance, we did not perform additional inferential analysis with the variables retained by the models and hence no odds ratios are presented here based on LASSO 
Table 4 LASSO model summary statistics and goodness-of-fit statistics in predicting all-cause ED visits in children and adolescents

\begin{tabular}{lllll}
\hline Model & lambda & Number of non-zero coefficients & In-sample deviance ratio & Out of sample deviance ratio \\
\hline Cross Validated & 0.0107871 & 113 & 0.066 & 0.0246 \\
Adaptive & 0.0016839 & 104 & 0.099 & -0.0448
\end{tabular}

models. Also, the coefficients selected by the two models are not presented and discussed here since the models did not have an adequate predictive performance for the coefficients to be usable in creating risk-scoring algorithms.

\section{Subgroup analysis}

Within a year of enrolment to the CAAMHPP program, $16.8 \%(95 \%$ CI $15.9,17.8)$ of children and adolescents visited the ED with mental health issues. The CV and adaptive LASSO models failed to perform well (poor discrimination and calibration) in predicting mental illness specific ED visits among children and adolescents (Supplementary Tables 1 and 2: Additional file 2). The sample comprised $75.3 \%$ adolescents, and $45 \%$ among them had an ED visit within a year. The two LASSO models did not demonstrate adequate performance in predicting all-cause ED visits among adolescents as well (Supplementary Tables 3 and 4: Additional file 2).

\section{Sensitivity analysis}

The median ACE score and interquartile range in the sample was 2 (IQR 1-4). We examined the ability of the cumulative scores in predicting ED visits in our study and found that the performance was inadequate $($ AUC < 0.7) (Supplementary Tables 1 and 2: Additional file 3 ). The predictive ability of threat-based and deprivation-based adversities from the ACEs checklist examined as two separate models also failed to demonstrate clinically meaningful predictive performance as evidenced by AUC $<0.7$ (Supplementary Tables 3 to 6: Additional file 3).

\section{Discussion}

\section{Summary}

The study's objective was to explore whether predictive models using machine learning approaches could predict ED visits with sufficient accuracy to move forward with developing a decision support tool for clinicians to stratify children and adolescents into various risk categories.
The bivariate analysis in the full sample showed that most of the ACE items were significantly associated with the subsequent ED visit. The statistically significant odds ratios ranged from 1.2 to 1.7; for instance, the odds ratio associated with sexual abuse was 1.72 (95\% CI 1.41,2.10), with physical abuse was $1.56(95 \%$ CI $1.35,1.81)$ and with parental divorce was 1.23 (95\% CI 1.11,1.37). This finding is consistent with the previous literature, which has reported that ACEs are associated with higher health service utilization $[10,11,13,14]$. The strong association of the adversities with ED visits indicates the important role of ACEs in health care utilization, even if they do not accurately support individual level predictions. Other demographic and clinical characteristics such as being female and having a mental health diagnosis were associated with frequent ED visits among children and adolescents in the study, which is also similar to the findings of other studies $[19,20]$.

However, the prediction of outcomes at the individual level is much more challenging than comparing frequencies according to statistical significance. The LASSO logistic regression models employing two different lambda selection methods, namely cross-validated and adaptive, failed to perform well in the database in predicting ED visits. The AUCs never reached 0.80 in the validation sample to be considered good discriminatory performance, and the ROC curves were closer to the reference line. This means that the models did not discriminate well between children who did and did not visit ED. The maximum sensitivity achieved was $37.8 \%$, which means that the model identified only $37.8 \%$ of the children who actually visited ED, and the PPV was 49.6\%, which means that of those who were predicted or flagged by the model to have an ED visit in future, not even $50 \%$ of them had an ED visit and more than $50 \%$ would be false positive. A good calibration performance is more important when the purpose of prediction is prognostic [35]. Concordance between observed and predicted rates of ED visits was not achieved in the various risk categories $(<25 \%, 25-49 \%, 50-74 \%,>75 \%)$ to be considered a

Table 5 Discrimination performance of the LASSO logistic regression models in the validation dataset in predicting all-cause ED visits among children and adolescents

\begin{tabular}{|c|c|c|c|c|c|c|c|}
\hline \multirow[t]{2}{*}{ Model } & \multirow{2}{*}{$\begin{array}{l}\text { Training data } \\
\text { AUC }\end{array}$} & \multicolumn{6}{|c|}{ Validation data } \\
\hline & & AUC & Sensitivity\% & Specificity\% & PPV\% & NPV\% & Overall \% \\
\hline Cross Validated & 0.680 & 0.610 & 29.2 & 82.2 & 52.5 & 63.3 & 60.9 \\
\hline Adaptive & 0.698 & 0.604 & 37.8 & 74.1 & 49.6 & 63.9 & 59.5 \\
\hline
\end{tabular}



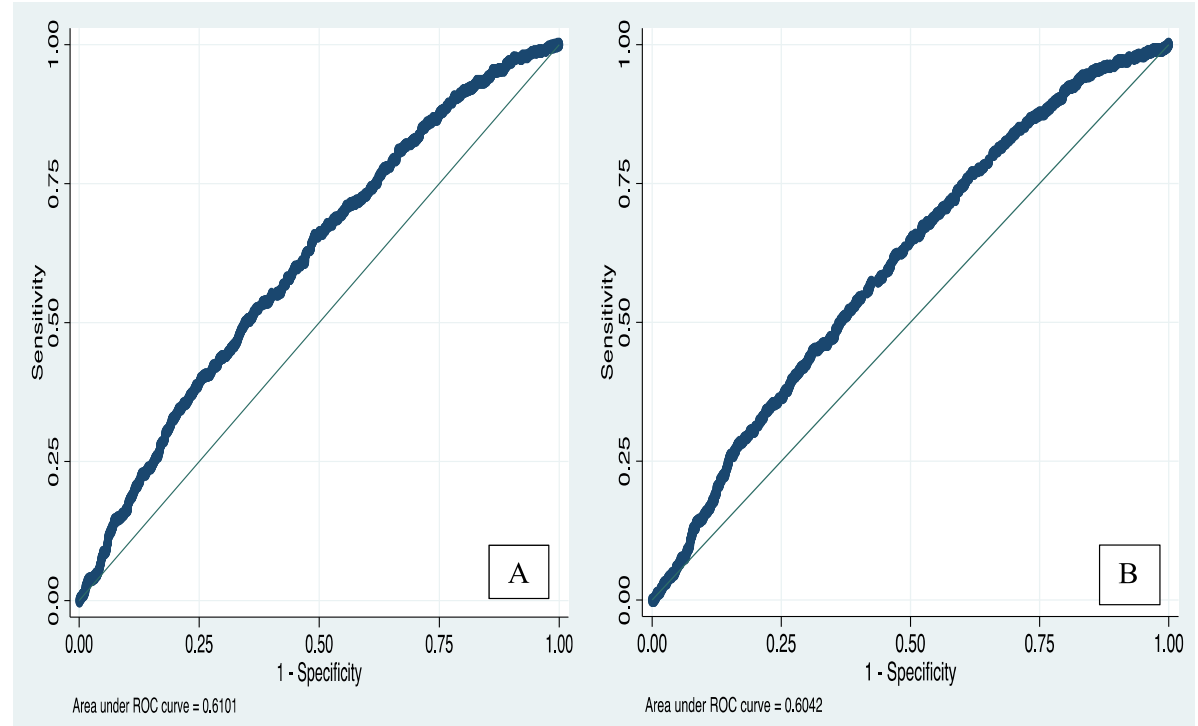

Fig. 2 ROC curves of the LASSO logistic regression models in validation dataset in predicting all-cause ED visits among children and adolescents (A) CV (B) Adaptive

good calibration performance, where there was evidence of over-triage in the low-risk category and under-triage in the high-risk category. The poor calibration was evident in the graphs of predicted versus observed proportions, where the fitted calibration curves deviated substantially from the reference/perfect prediction line. The out-of-sample deviance ratios were far less than 1 , demonstrating their inability to perform well in the validation dataset. Hence, the ACE items in the ACE checklist did not have a useful degree of ability to identify children and youth at risk of requiring a visit to the ED within a year of presenting to the mental health services and thus cannot be applied clinically to stratify risk of ED visits.

The subgroup analysis showed that ACEs did not perform well in predicting all-cause ED visits among adolescents and predicting mental-illness-related ED visits among children and adolescents. Furthermore, the prediction models did not show promising results in predicting ED visits in an earlier period, i.e., within 6 months of enrolment to the program (results not shown). Similarly, the use of cumulative ACE scores and different dimensions of adversities (threat and deprivation) also did not improve the predictions. The adaptive LASSO prediction model using ACE scores had an AUC of 0.621 with a sensitivity of $38.9 \%$. The model with threat-based adversities had an AUC of 0.608 (sensitivity $38.1 \%$ ), and that with deprivation-based adversities had an AUC of 0.607 (sensitivity 38.6\%). These discrimination properties were similar to those achieved using each ACE item separately in the model (AUC 0.604 , sensitivity $37.8 \%$ ). There was non-concordance between observed and predicted rates of ED visits in all the models. This suggests that the predictive performances of the various approaches of modeling adverse childhood experiences used in the study were equivalent

Table 6 Calibration matrix of the LASSO regression models in the validation dataset in predicting all-cause ED visits among children and adolescents

\begin{tabular}{llll}
\hline Model & Predicted probability of ED visit \% & Observed ED visit \% (95\%Cl) & $\begin{array}{l}\text { Risk stratification capacity } \\
\text { N (\%) }\end{array}$ \\
\hline Cross Validated & $0-24$ & $15.0(9.2,23.5)$ & $393(6.44)$ \\
& $25-49$ & $38.8(35.9,41.7)$ & $4330(70.98)$ \\
& $50-74$ & $52.5(47.2,57.8)$ & $1364(22.36)$ \\
& $75-100$ & $50.0(4.0,96.0)$ & $13(0.21)$ \\
Adaptive & $0-24$ & $21.7(16.6,27.8)$ & $957(15.69)$ \\
& $25-49$ & $39.8(36.5,43.1)$ & $3296(54.03)$ \\
& $50-74$ & $50.8(45.8,55.7)$ & $1592(26.10)$ \\
\end{tabular}




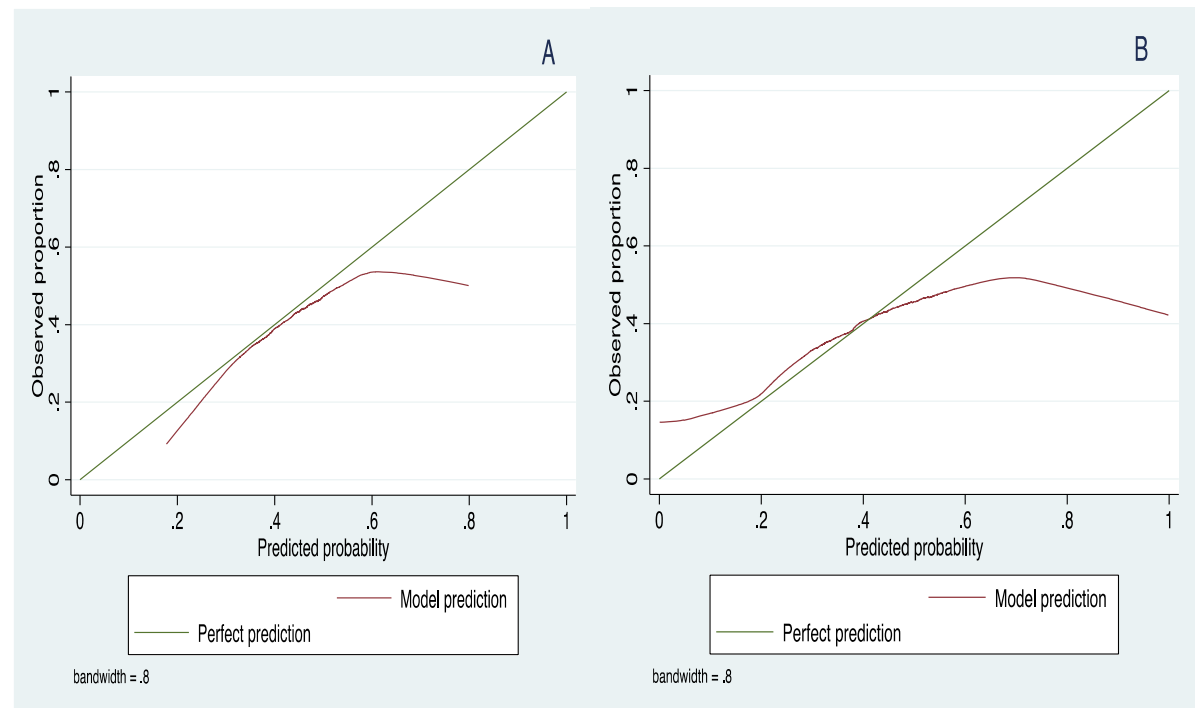

Fig. 3 Calibration plots of the LASSO models in validation dataset in predicting all-cause ED visits among children and adolescents; (A) CV (B) Adaptive

in predicting ED visits among children and adolescents with pre-existing mental health issues.

\section{Interpretation}

If the prediction model had performed well, it might have been sufficiently accurate for the clinicians and policymakers to early identify which of the patients would be visiting the ED for physical or mental health complaints in the future. This valuable information might have supported the development of decision support tools or improvements to care pathways in outpatient services to prevent ED visits through improved patient education, discharge planning, and follow-up care. Also, there are many resources required to collect and store data in a clinical setting and collecting ACEs data has challenges due to the sensitive nature of the subject. Hence, the policymakers and care providers need to know whether the data on ACEs should be routinely collected or not by clinicians. The prediction models are not intended to replace clinicians but support them in their clinical judgment by combining knowledge of machine learning with knowledge about childhood exposure to adversity [55]. The prediction models formulated by incorporating a wide range of predictors provide them additional information that they might not have access to or might not have enough time and resources to take into account while formulating their decisions, making the care pathways more efficient. Health policymakers and administrators are always looking for ways to use scarce resources efficiently, and prediction models could help do so [31]. However, such prediction models should be developed and used with caution because the use of poor-performing models to predict events and guide decisions may, in turn, harm the clients as well as waste the scarce resources.

While the predictive models developed in this project ultimately did not predict ED visits with sufficient accuracy to be clinically useful, research of the type presented here is important to the current trend in the direction of precision health. The situations in which routinely collected data may have value for prediction and prediction may have value for clinical management are not well understood and need to be explored. The results presented here suggest that despite statistical associations of the ACEs with relevant clinical outcomes, the addition of ACE items provide insufficient data to predict ED visits at the individual level, which lacks utility for clinical use. Future studies, including a more extensive set of predictors such as the severity of mental illness, a wide range of other childhood adversities with their frequency and severity, age at exposure, other sociodemographic variables such as family income, parental education status, social support, resilience and other important clinical or behavioral information, are required which might improve the predictive ability of the machine learning models. These variables are reported to be associated with increased health service utilization $[11,19,20]$. However, a significant association with the outcome is not exclusive to being included as potential predictors in a prediction model.

The RAIS database contains a limited range of other variables that could improve the models' predictive ability. Hence, child and adolescent mental health programs should consider collecting more information about childhood exposure to adversities. A recent Canadian 
study has suggested that in addition to the ACE items, expanded ACEs such as household gambling problems, spanking, peer victimization, and neighborhood safety factor well with child maltreatment and household dysfunction and are associated with poor physical and mental health [56]. Collecting data on these emerging adversities through the health system and using them to predict health outcomes could also be the future direction.

Our results do not suggest that ACEs are not useful or not important for prediction at all. Although the vast majority of literature which reports child maltreatment as predictors of adverse health have merely examined associations $[25,57,58]$, few studies have used ACEs to predict health outcomes using a prediction modeling approach. The limited studies have found that history of child abuse and household dysfunctions predict adverse mental health and psychosocial disadvantages $[35,59,60]$, but not much is published about predicting health service utilization. The outcome, i.e., ED visits, maybe inherently less predictable as it may depend on chance events such as injuries, illness, or psychosocial circumstances such as homelessness and poverty [19]. Also, ACEs might be used for many purposes other than predicting ED visits. These include health risk behaviors, chronic diseases, and hospitalizations, and future studies should look into such predictions. The ability to predict and mitigate other proximal adverse health effects of ACEs such as smoking, alcohol and illicit drug use may also benefit children and adolescents to prevent future health emergencies and unplanned need for acute health care services [47].

The population under study was a highly selected subgroup (i.e., children who are already seeking mental health services), which is already a high-risk cohort for ED visits. Future studies should also explore whether ACE items could predict ED visits among children and adolescents from the general population. The ACE checklist in itself has its limitations in terms of limited item coverage and has not been validated [61]. Hence, the inability of the ACE checklist to demonstrate good predictive ability does not suggest that other validated tools that measure childhood adversities, such as Childhood Trauma Questionnaire (CTQ), Conflict Tactic Scales, may not be useful in predicting health outcomes.

The LASSO models' poor performance in the dataset we used may not mean that machine learning lacks utility for predicting health outcomes. It has been reported that machine learning techniques demonstrated good performance in predicting adverse health that may develop after child abuse [59]. Most of our predictors were categorical variables, and some reports suggest that machine learning might perform better in prediction if there are a large number of continuous variables than binary or factor variables [47]. Furthermore, it is not surprising that the small number of variables we used were not adequately predictive of the outcome $[47,48]$. We also used only one type of machine learning approach (i.e., LASSO regression) for predicting our outcome, which assumes a linear relationship between predictors and the outcome. Future studies should assess whether the ACE items predict future ED visits by employing other machine learning approaches such as neural networks, gradient boosted trees, and support vector machines, which can take into account the nonlinearities in the relationship between the predictors and the outcome better, if any [47]. The performance of the models may also improve with a bigger sample size [48], which in our case can be achieved by analyzing a more extended period of data.

The study has some strengths and limitations. This study's relevance is strong because childhood adversities are a significant public health problem, leading to various health issues throughout the lifespan and contributing to an increased burden on health services and considerable healthcare costs. Prediction modeling is a new and advanced method of incorporating routinely available data into precision health applications. This study used routinely collected ACE data to predict future ED visits among children and adolescents for the first time in Alberta Health Services. The study data came from patients from one geographic region of Alberta; the prediction modeling results might not be generalizable to other populations due to differences in the prevalence of the outcome or inclusion and exclusion criteria of the patient population. However, the generalizability has low priority in prediction modeling because the models are generally optimized for a particular clinical setting to aid in clinical judgment. The random split sample for training and validation and cross-validation method applied in the LASSO models might have replication instability such that the different random subsets of data might lead to differences in the prediction performance measures [45]. However, the difference was not big enough to change the interpretation of the results in this study. The parental reports of ACEs might have been biased (potentially under-reported) due to the stigma attached to the adversities, the fear of child welfare reporting, or being the perpetrators themselves. However, the ACEs were still significantly associated with ED visits. Hence, we do not believe that the heterogeneity of the informants on ACEs would have affected the performance of prediction models. As described above, an indicator of missing data was included as a predictor in the modeling and contributed to the predictive algorithm. However, indicators for actual events rather than missing data indicators would have improved the predictive performance of the model to a greater extent. Therefore, missing data remains a limitation of this study. Although the objective of the study was to assess the predictive ability of ACE checklist, it is 
important to acknowledge that the checklist addresses a very limited range of possible childhood adversities that are more relevant to the population under study. For instance, the sample was predominantly urban, and it has been reported that urban youth are at increased risk of exposure to violence in community, both direct or indirect victimization, and the exposure would be higher if they belonged to poor and disadvantaged households and communities [62]. These exposures may increase the risk of adverse health and behavioural outcomes and might prove to be significant predictors of poor health and health service utilization in future studies. To further the goal of prediction, it will be important that such adversities be collected and recorded in accessible data sources.

\section{Conclusion}

The ACE items from the ACE checklist were strongly associated with ED visits. However, the ACEs, along with the clinical and demographic information available from the RAIS database and their various combinations, did not demonstrate acceptable performance in predicting ED visits within a year of enrolment in an intensive outpatient mental health service program, and hence was not sufficient for application in clinical practice. This result was obtained using a state-of-the-art machine learning model with known strong predictive performance. We require good sensitivity and PPV in the model to correctly identify children who need additional interventions and not deploy resources inappropriately. The findings suggest that ACEs are important risk factors associated with health service utilization but cannot be viewed as deterministic causes of health events. Other machine learning methods or statistical models that include additional important variables such as the severity of mental illness, frequency and severity of ACEs, peer victimization, neighborhood safety, social support, and resilience may produce better performing prediction models. Such models can aid in clinical judgment, implementation of care pathways, and patient education to prevent ED visits. However, they would require a large quantity of additional routinely collected data, especially childhood adversity data in greater depth and breadth.

\section{Abbreviations}

ACEs: Adverse Childhood Experiences; ED: Emergency Department; AHS: Alberta Health Services; CAAMHPP: Child and Adolescent Addiction and Mental Health and Psychiatry Program; RAIS: Regional Access and Intake System; NACRS: National Ambulatory Care and Reporting System;

LASSO: Least Absolute Shrinkage and Selection Operator; AUC: Area Under the receiver Operating Characteristics Curve; CV: Cross-validated; PPV: Positive Predictive Value

\section{Supplementary Information}

The online version contains supplementary material available at https://doi. org/10.1186/s12874-021-01392-w.
Additional file 1. Coefficients selected by the LASSO models.

Additional file 2. Results of subgroup analyses.

Additional file 3. Results of sensitivity analyses.

\section{Acknowledgments}

The authors thank Alberta Health Services (AHS) and Alberta Strategy for Patient-Oriented Research SUPPORT Unit (AbSPORU) for providing access to the databases used for analysis in the study.

\section{Authors' contributions}

A.B., S.B.P. conceived and designed the study, guided by discussions with G.D., S.C.T., and A.G.M.B. Data management, data analysis, and interpretation of results, and drafting the manuscript was performed by A.B., guided and supervised by S.B.P. The data access was facilitated by B.M. and J.P., who also supported data management and provided intellectual content to the study. All authors assisted with preparing this manuscript by providing conceptual and analytical inputs and read and approved the final draft.

\section{Funding}

A.B. is supported by a Ph.D. studentship award from Alberta Innovates Health Solutions (AlHS Funding Reference: 201810552). S.B.P. holds the Cuthbertson and Fischer Chair in Pediatric Mental Health. The funding agencies had no role in the design of the study, data access, analysis, interpretation of results and writing the manuscript.

\section{Availability of data and materials}

The datasets generated and/or analysed during the current study are not publicly available due to limitations of ethical approval involving the patient data and anonymity but are available from the corresponding author on reasonable request.

\section{Declarations}

\section{Ethics approval and consent to participate}

The University of Calgary Conjoint Health Research Ethics Board (CHREB) approved all the experimental protocols (REB18-2097). The study was conducted under the auspices of University of Calgary, under the institution's research protocols and procedures.

\section{Consent for publication}

Not applicable.

\section{Competing interests}

The authors declare that they have no competing interests.

\section{Author details}

'Department of Community Health Sciences, Cumming School of Medicine, University of Calgary, 3280 Hospital Drive NW, Calgary, AB T2N4Z6, Canada.

${ }^{2}$ Mathison Centre for Research \& Education, University of Calgary, 3280 Hospital Drive NW, Calgary, AB T2N4Z6, Canada. ${ }^{3}$ Faculty of Social Work, University of Calgary, 2500 University Dr NW, Calgary, AB T2N 1N4, Canada. ${ }^{4}$ Addiction and Mental Health, Alberta Health Services- Calgary Zone Calgary, AB Canada . ${ }^{5}$ Department of Psychiatry, Cumming School of Medicine, University of Calgary, 2500 University Dr NW, Calgary, AB T2N 1N4, Canada. ${ }^{6}$ Department of Pediatrics, Cumming School of Medicine, University of Calgary, 2500 University Dr NW, Calgary, AB T2N 1N4, Canada.

Received: 29 January 2021 Accepted: 4 September 2021

Published online: 25 September 2021

\section{References}

1. Felitti VJ, Anda RF, Nordenberg D, Williamson DF, Spitz AM, Edwards V, et al. Relationship of childhood abuse and household dysfunction to many of the leading causes of death in adults. The adverse childhood experiences (ACE) study. Am J Prev Med. 1998;14(4):245-58. https://doi.org/10.1016/S0749-3 797(98)00017-8.

2. McDonald S, Kingston D, Bayrampour H, Tough S. Adverse childhood experiences in Alberta, Canada: a population based study. Med Res Arch. 2015;3(3). https://doi.org/10.18103/mra.v0i3.142. 
3. Hall J, Porter L, Longhi D, Becker-Green J, Dreyfus S. Reducing adverse childhood experiences (ACE) by building community capacity: a summary of Washington family policy council research findings. J Prev Interv Community. 2012;40(4):325-34. https://doi.org/10.1080/10852352.2 012.707463

4. Hughes K, Lowey H, Quigg Z, Bellis MA. Relationships between adverse childhood experiences and adult mental well-being: results from an English national household survey. BMC Public Health. 2016;16(1):222. https://doi. org/10.1186/s12889-016-2906-3

5. Bellis MA, Hughes K, Leckenby N, Hardcastle K, Perkins C, Lowey H. Measuring mortality and the burden of adult disease associated with adverse childhood experiences in England: a national survey. J Public Health. 2015;37(3):445-54. https://doi.org/10.1093/pubmed/fdu065.

6. Substance Abuse and Mental Health Services Administration. SAMHSA's concept of trauma and guidance for a trauma-informed approach. Rockville: HHS Publication No.(SMA) 14-4884; 2014.

7. Pathak P, Grimes K. Adverse childhood experiences (ACE) assessment in clinical practice: a pediatric integrated care model; 2019. Am Acad Pediatrics.

8. Oral R, Ramirez M, Coohey C, Nakada S, Walz A, Kuntz A, et al. Adverse childhood experiences and trauma informed care: the future of health care. Pediatr Res. 2016;79(1):227-33. https://doi.org/10.1038/pr.2015.197.

9. Cawthorpe D, Marriott B, Paget J, Moulai I, Cheung S. Relationship between adverse childhood experience survey items and psychiatric disorders. Perm J. 2018;22. https://doi.org/10.7812/TPP/18-001.

10. Chartier MJ, Walker JR, Naimark B. Separate and cumulative effects of adverse childhood experiences in predicting adult health and health care utilization. Child Abuse Negl. 2010;34(6):454-64. https://doi.org/10.1016/j. chiabu.2009.09.020.

11. Tossone K, Jefferis E, Bhatta MP, Bilge-Johnson S, Seifert P. Risk factors for rehospitalization and inpatient care among pediatric psychiatric intake response center patients. Child Adolesc Psychiatry Ment Health. 2014;8(1):27. https://doi.org/10.1186/1753-2000-8-27.

12. Hargreaves MK, Mouton CP, Liu J, Zhou YE, Blot WJ. Adverse childhood experiences and health care utilization in a low-income population. J Health Care Poor Underserved. 2019;30(2):749-67. https://doi.org/10.1353/hpu.2019. 0054.

13. Bellis M, Hughes K, Hardcastle K, Ashton K, Ford K, Quigg Z, et al. The impact of adverse childhood experiences on health service use across the life course using a retrospective cohort study. J Health Serv Res Policy. 2017; 22(3):168-77. https://doi.org/10.1177/1355819617706720.

14. Alcalá HE, Valdez-Dadia A, von Ehrenstein OS. Adverse childhood experiences and access and utilization of health care. J Public Health. 2018; 40(4):684-92. https://doi.org/10.1093/pubmed/fdx155.

15. Chartier M, Walker J, Naimark B. Childhood abuse, adult health, and health care utilization: results from a representative community sample. Am J Epidemiol. 2007;165(9):1031-8. https://doi.org/10.1093/aje/kwk113.

16. Koskenvuo K, Koskenvuo M. Childhood adversities predict strongly the use of psychotropic drugs in adulthood: a population-based cohort study of 24 284 Finns. J Epidemiol Community Health. 2015;69(4):354-60. https://doi. org/10.1136/jech-2014-204732.

17. Enard KR, Ganelin DM. Reducing preventable emergency department utilization and costs by using community health workers as patient navigators. J HealthcManag. 2013;58(6):412.

18. Yogendran LV, Hany M, Chaucer B, Pasha S, Kaur S, Janusz C. The effects of Abuse and substance use on emergency department visits and hospital admissions among child and adolescent patients; 2018. Am Acad Pediatrics.

19. Slankamenac K, Heidelberger R, Keller DI. Prediction of recurrent emergency department visits in patients with mental disorders. Front Psychiatry. 2020; 11. https://doi.org/10.3389/fpsyt.2020.00048.

20. Niedzwiecki MJ, Sharma PJ, Kanzaria HK, McConville S, Hsia RY. Factors associated with emergency department use by patients with and without mental health diagnoses. JAMA Netw Open. 2018;1(6):e183528-e.

21. Centers for Disease Control and Prevention. Adverse childhood experiences: Looking at how ACEs affect our lives and society. Atlanta: Centers for Disease Control and Prevention (CDC); 2012. Retrieved from http:// vetoviolence.cdc.gov.

22. Wu J, Grannis SJ, Xu H, Finnell JT. A practical method for predicting frequent use of emergency department care using routinely available electronic registration data. BMC Emerg Med. 2016;16(1):12. https://doi.org/1 0.1186/s12873-016-0076-3.
23. Analytica R. The impact of the CFHI health care collaborations and initiatives; 2013.

24. Alberta Health Services. Alberta Health Services Annual Report 2018-2019 2019.

25. Jackson Y, Cushing CC, Gabrielli J, Fleming K, O'Connor BM, Huffhines L. Child maltreatment, trauma, and physical health outcomes: the role of abuse type and placement moves on health conditions and service use for youth in foster care. J Pediatr Psychol. 2016;41(1):28-36. https://doi.org/10.1 093/jpepsy/jsv066.

26. Das LT, Abramson EL, Stone AE, Kondrich JE, Kern LM, Grinspan ZM. Predicting frequent emergency department visits among children with asthma using EHR data. Pediatr Pulmonol. 2017;52(7):880-90. https://doi. org/10.1002/ppul.23735

27. Canadian Institute for Health Information. All-cause readmission to acute care and return to the emergency department. Health System Performance. 2012

28. Zarse EM, Neff MR, Yoder R, Hulvershorn L, Chambers JE, Chambers RA. The adverse childhood experiences questionnaire: two decades of research on childhood trauma as a primary cause of adult mental illness, addiction, and medical diseases. Cogent Med. 2019;6(1):1581447. https://doi.org/10.1 080/2331205X.2019.1581447.

29. Adhikari K, Patten SB, Williamson T, Patel AB, Premji S, Tough S, et al. Does neighborhood socioeconomic status predict the risk of preterm birth? A community-based Canadian cohort study. BMJ Open. 2019;9(2):e025341. https://doi.org/10.1136/bmjopen-2018-025341.

30. Samuels-Kalow M, Peltz A, Rodean J, Hall M, Alpern ER, Aronson PL, et al. Predicting low-resource-intensity emergency department visits in children. Acad Pediatr. 2018;18(3):297-304. https://doi.org/10.1016/j.acap.2017.12.012.

31. Sanderson M, Bulloch AG, Wang J, Williams KG, Williamson T, Patten SB. Predicting death by suicide following an emergency department visit for parasuicide with administrative health care system data and machine learning. EClinicalMedicine. 2020;100281. https://doi.org/10.1016/j.eclinm.202 0.100281 .

32. Clausen AN, Aupperle RL, Yeh H-W, Waller D, Payne J, Kuplicki R, et al. Machine learning analysis of the relationships between gray matter volume and childhood trauma in a transdiagnostic community-based sample. Biol Psychiatry Cogn Neurosci Neuroimaging. 2019;4(8):734-42. https://doi.org/1 0.1016/j.bpsc.2019.03.001.

33. Goto T, Camargo CA, Faridi MK, Freishtat RJ, Hasegawa K. Machine learningbased prediction of clinical outcomes for children during emergency department triage. JAMA Netw Open. 2019;2(1):e186937-e.

34. Saxe GN, Ma S, Ren J, Aliferis C. Machine learning methods to predict child posttraumatic stress: a proof of concept study. BMC Psychiatry. 2017;17(1): 223. https://doi.org/10.1186/s12888-017-1384-1.

35. Latham RM, Meehan AJ, Arseneault L, Stahl D, Danese A, Fisher HL. Development of an individualized risk calculator for poor functioning in young people victimized during childhood: a longitudinal cohort study. Child Abuse Negl. 2019;98:104188. https://doi.org/10.1016/j.chiabu.2019.104188.

36. Grinspan ZM, Patel AD, Hafeez B, Abramson EL, Kern LM. Predicting frequent emergency department use among children with epilepsy: a retrospective cohort study using electronic health data from 2 centers. Epilepsia. 2018;59(1):155-69. https://doi.org/10.1111/epi.13948.

37. Soril LJ, Leggett LE, Lorenzetti DL, Noseworthy TW, Clement FM. Characteristics of frequent users of the emergency department in the general adult population: a systematic review of international healthcare systems. Health Policy. 2016;120(5):452-61. https://doi.org/10.1016/j.hea Ithpol.2016.02.006.

38. Poole S, Grannis S, Shah NH. Predicting emergency department visits. AMIA Summits Transl Sc Proc. 2016;2016:438.

39. Yang C, Delcher C, Shenkman E, Ranka S. Machine learning approaches for predicting high utilizers in health care. International Conference on Bioinformatics and Biomedical Engineering. Denmark: Springer; 2017.

40. Fonti $V$. Research paper in business analytics: feature selection with LASSO. Amsterdam: VU Amsterdam; 2017.

41. Alberta Health Services. User Manual for RAIS: Regional Access \& Intake System. Data Management, Decision Support Team Addiction and Mental Health Alberta Health Services -Calgary Zone. 2018.

42. Alberta Health Services. Child \& Adolescent Addiction, Mental Health and Psychiatry Program (CAAMHPP). Adverse Childhood Experiences (ACE) / Trauma Informed Resource Guide. 2016. 
43. Rahman A, Perri A, Deegan A, Kuntz J, Cawthorpe D. On becoming traumainformed: role of the adverse childhood experiences survey in tertiary child and adolescent mental health services and the association with standard measures of impairment and severity. Perm J. 2018;22. https://doi.org/10. 7812/TPP/17-054.

44. StataCorp L. Stata statistical software: release 16. TX: College Station; 2019.

45. Shipe ME, Deppen SA, Farjah F, Grogan EL. Developing prediction models for clinical use using logistic regression: an overview. J Thorac Dis. 2019; 11(Suppl 4):S574-84. https://doi.org/10.21037/jtd.2019.01.25

46. Puterman E, Weiss J, Hives BA, Gemmill A, Karasek D, Mendes WB, et al. Predicting mortality from 57 economic, behavioral, social, and psychological factors. Proc Natl Acad Sci. 2020;117(28):16273-82. https://doi.org/10.1073/ pnas.1918455117.

47. Desai RJ, Wang SV, Vaduganathan M, Evers T, Schneeweiss S. Comparison of Machine Learning Methods With Traditional Models for Use of Administrative Claims With Electronic Medical Records to Predict Heart Failure Outcomes. JAMA Netw Open. 2020;3(1):e1918962-e.

48. Tate AE, McCabe RC, Larsson H, Lundström S, Lichtenstein P, Kuja-Halkola R. Predicting mental health problems in adolescence using machine learning techniques. PLoS One. 2020;15(4):e0230389. https://doi.org/10.1371/journal. pone.0230389.

49. StataCorp L. STATA LASSO reference manual release 16. TX: College Station; 2019.

50. Mall S, Mortier P, Taljaard L, Roos J, Stein DJ, Lochner C. The relationship between childhood adversity, recent stressors, and depression in college students attending a south African university. BMC Psychiatry. 2018;18(1):110. https://doi.org/10.1186/s12888-017-1583-9.

51. Berger $M$, Schmid $M$. Semiparametric regression for discrete time-to-event data. Stat Model. 2018;18(3-4):322-45. https://doi.org/10.1177/1471082X 7748084.

52. Flaherty EG, Thompson R, Dubowitz H, Harvey EM, English DJ, Proctor LJ, et al. Adverse childhood experiences and child health in early adolescence. JAMA Pediatr. 2013;167(7):622-9. https://doi.org/10.1001/jamapediatrics.2 013.22 .

53. McLaughlin KA, Green JG, Gruber MJ, Sampson NA, Zaslavsky AM, Kessler RC. Childhood adversities and first onset of psychiatric disorders in a national sample of US adolescents. Arch Gen Psychiatry. 2012;69(11):115160. https://doi.org/10.1001/archgenpsychiatry.2011.2277.

54. McLaughlin KA, Weissman D, Bitrán D. Childhood adversity and neura development: a systematic review. Annu Rev Dev Psychol. 2019;1(1):277312. https://doi.org/10.1146/annurev-devpsych-121318-084950.

55. Gillingham P. Can predictive algorithms assist decision-making in social work with children and families? Child Abuse Rev. 2019;28(2):114-26. https://doi.org/10.1002/car.2547.

56. Afifi TO, Salmon S, Garcés I, Struck S, Fortier J, Taillieu T, et al. Confirmatory factor analysis of adverse childhood experiences (ACEs) among a community-based sample of parents and adolescents. BMC Pediatr. 2020; 20(1):178. https://doi.org/10.1186/s12887-020-02063-3.

57. Moore SM. Child maltreatment and physical health in emerging adulthood: relationships with physical activity and health risk behaviors; 2019.

58. Widom CS, Czaja SJ, Bentley T, Johnson MS. A prospective investigation of physical health outcomes in abused and neglected children: new findings from a 30-year follow-up. Am J Public Health. 2012;102(6):1135-44. https:// doi.org/10.2105/AJPH.2011.300636.

59. Gokten ES, Uyulan C. Prediction of the Development of Depression and Post-traumatic Stress Disorder in Sexually Abused Children using a Random Forest Classifier. J Affect Dis. 2020;279:256-65.

60. Berman IS, Petretric P, Bridges AJ. Beyond child maltreatment: the incremental value of household dysfunction in the prediction of negative beliefs and internalizing symptoms in women. J Am Coll Heal. 2019;69(5):19.

61. McLennan JD, MacMillan HL, Afifi TO. Questioning the use of adverse childhood experiences (ACEs) questionnaires. Child Abuse Negl. 2020;101: 104331. https://doi.org/10.1016/j.chiabu.2019.104331.

62. Seal D, Nguyen A, Beyer K. Youth exposure to violence in an urban setting. Urban Stud Res. 2014;2014:1-11. https://doi.org/10.1155/2014/368047.

\section{Publisher's Note}

Springer Nature remains neutral with regard to jurisdictional claims in published maps and institutional affiliations.

Ready to submit your research? Choose BMC and benefit from:

- fast, convenient online submission

- thorough peer review by experienced researchers in your field

- rapid publication on acceptance

- support for research data, including large and complex data types

- gold Open Access which fosters wider collaboration and increased citations

- maximum visibility for your research: over $100 \mathrm{M}$ website views per year

At BMC, research is always in progress.

Learn more biomedcentral.com/submissions 